\title{
Orbital metastasis of endocervical stromal sarcoma: a rare tumor and an uncommon metastasis
}

\author{
Mehmet Metin Seker ${ }^{1}$, Ali Ugur Uslu², Hatice Ozer ${ }^{3}$, Ayse Seker ${ }^{4}$, Turgut Kacan ${ }^{1}$, Nalan Babacan ${ }^{1}$, \\ Handan Aker ${ }^{3}$, Sahande Elagoz ${ }^{3}$
}

${ }^{1}$ Department of Medical Oncology, Medical Faculty, Cumhuriyet University, Sivas, Turkey

${ }^{2}$ Department of Internal Medicine, Medical Faculty, Cumhuriyet University, Sivas, Turkey

${ }^{3}$ Department of Pathology, Medical Faculty, Cumhuriyet University, Sivas, Turkey

${ }^{4}$ Ibni Sina Public Health Care Center, Sivas, Turkey

\begin{abstract}
Endocervical stromal sarcoma (ECSS) is a very rare uterine sarcoma. The most common presentation is pelvic mass and vaginal bleeding. The mainstay of treatment is surgery. There is no consensus on the adjuvant treatment. Relapses are usually in the pelvic and abdominal regions. To a lesser extent, lung, liver and bone metastases may be seen.

A 46-year-old woman had total abdominal hysterectomy (TAH) with bilateral salpingo-oophorectomy (BSO) performed due to endometrial polyp and leiomyoma. Six months after the TAH-BSO, she was admitted to the hospital with cough and hemoptysis. A thoracic mass was detected, and a biopsy was done. The diagnosis was low-grade ECSS metastasis. One week after thoracotomy, she was admitted to the hospital with loss of vision in the left eye. An orbital mass was detected with magnetic resonance imaging. Endometrial and cervical pathology preparations were reassessed and were compatible with ECSS. We performed mammography, thorax, and abdomen and cranial imaging to rule out other malignancies that may cause lung and orbital metastasis. Partial remission was achieved with systemic chemotherapy and orbital radiotherapy. Orbital metastasis may be seen in ECSS patients. Although we have less knowledge about the choice of chemotherapeutic agents, ifosfamide and doxorubicin are effective in treating ECSS.
\end{abstract}

Key words: endocervical stromal sarcoma, metastasis, orbital.

\section{Introduction}

Sarcomas constitute less than $1 \%$ of all uterine and cervical malignancies. Endocervical stromal sarcoma (ECSS) is a very rare uterine sarcoma [1]. To date there are only 17 patients in the literature. The most common presentation is pelvic mass and vaginal bleeding. The mainstay of the treatment is surgery. Although adjuvant radiation therapy and chemotherapy have been applied to some patients, the survival benefit of radiation therapy and chemotherapy in the adjuvant setting is unclear. So there is no consensus on the adjuvant treatment [2, 3]. Relapses are usually in the pelvic and abdominal regions. To a lesser extent, lung, liver and bone metastases may be seen [4]. The choice of treatment in local recurrences is surgery or radiation therapy, but systemic chemotherapy is preferred in the presence of distant metastases. To date, orbital metastasis due to ECSS has not been presented.

\section{Case report}

A 46-year-old female patient was admitted one year ago to the hospital with vaginal bleeding. During gynecologic examination, an endocervical polyp was detected and excised. After pathologic examination, the diagnosis was mesenchymal stromal polyps. After 4 months, she was admitted to the hospital with vaginal bleeding again. Thus, a total abdominal hysterectomybilateral salpingo oophorectomy (TAH-BSO) was performed. The diagnosis was mesodermal stromal polyp, chronic cervicitis and leiomyoma after pathologic examination (Fig. 1A). Six months after TAH-BSO, she was admitted to hospital with cough and hemoptysis. On chest computed tomography, a $61 \mathrm{~mm} \times 54 \mathrm{~mm}$ lobulated contoured mass lesion was obliterating the lower lobe bronchus and surrounding the descending aorta; a biopsy was performed. The diagnosis was low-grade malign mesenchymal tumor with pathologic examina- 

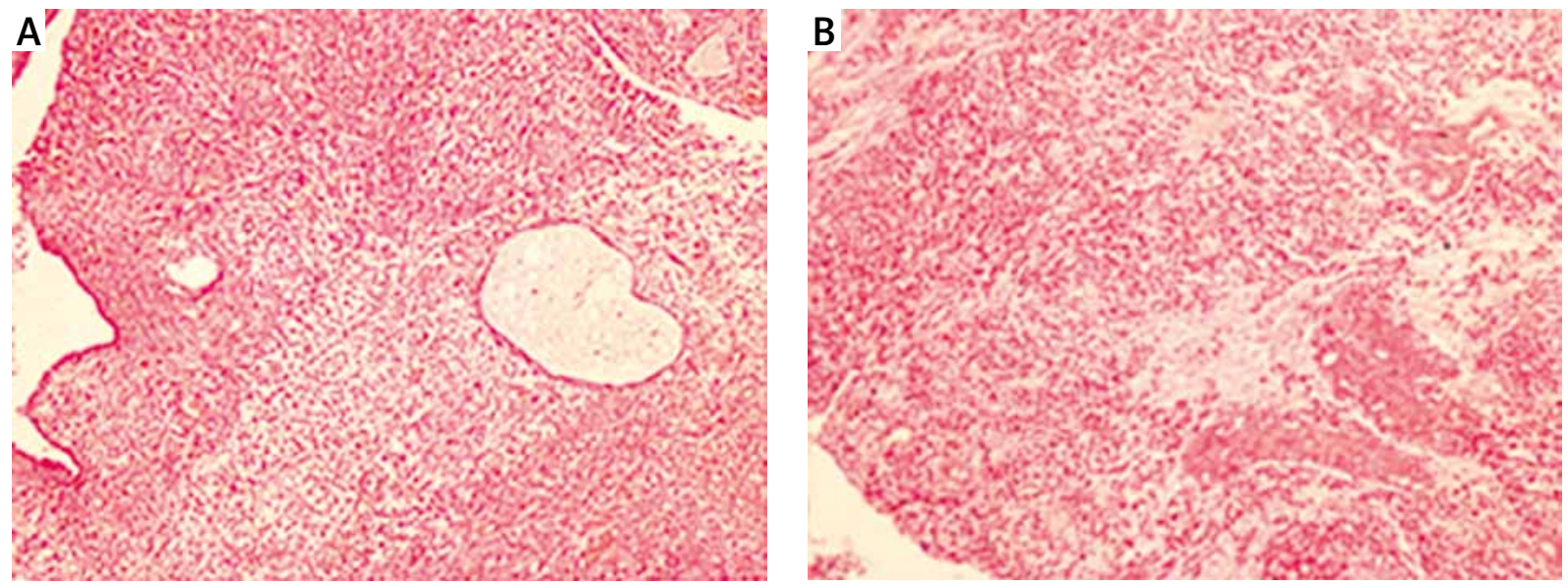

Fig. 1. Patient's cervical and lung biopsy specimens. A) Polypoid tumor below endocervical surface; stellate and spindle cells surrounding endocervical glands with edematous and myxoid background. Alternating hypercellular and hypocellular regions were noted (H\&E; magnification 100x). B) Sections of the lung mass exhibited morphologic features identical to those seen in uterine cervix. Proliferating hyalinized-walled blood vessels and high cellularity were also noted (H\&E; magnification 100x)

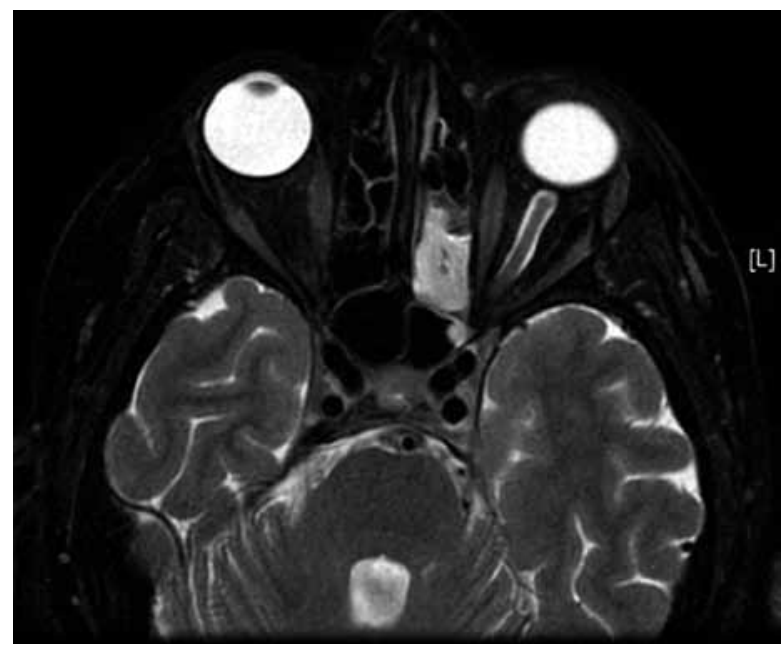

Fig. 2. Patient's orbital magnetic resonance imaging

tion. One week after biopsy, loss of vision in the left eye developed. Orbital magnetic resonance imaging was performed, and a $30 \mathrm{~mm} \times 20 \mathrm{~mm} \times 30 \mathrm{~mm}$ lesion was detected involving the orbital apex and posterior cavernous sinus; it was infiltrating the neural foramen and extending to the sphenoid sinus and ethmoid cells in the anteromedial (Fig. 2).

After detecting a malignant mesenchymal tumor in the patient's lung biopsy, the patient's endometrial and cervical tissue specimens were reexamined; the tumor comprised slightly to moderately pleomorphic stellate and spindle cells within an edematous or myxoid background (Fig. 1B). Alternating hypercellular and hypocellular regions were noted. In some areas, there was high mitotic activity. Proliferating hyalinized-walled blood vessels, myxoid background and hypercellularity were more pronounced, especially in the lung tumor tissue. Thus, we diagnosed ECSS. We performed a mammography and thorax, abdomen and cranial imaging to rule out other malignancies that may cause lung and orbital metastasis.

Because of the rapid progression, we preferred systemic chemotherapy as an initial treatment (ifosfamide $2500 \mathrm{mg} / \mathrm{m}^{2}$, d1-3; mesna $2500 \mathrm{mg} / \mathrm{m}^{2}$, d1-3; doxorubicin $60 \mathrm{mg} / \mathrm{m}^{2}, \mathrm{~d} 1$; q3 weeks). After three cycles of chemotherapy, our patient received orbital palliative radiotherapy. We had a partial response. Both the thoracic and orbital tumors regressed, and visual activity improved, even if it was blurred.

\section{Discussion}

The vast majority of cervical malignancies are squamous cell carcinomas. Endocervical stromal sarcoma accounts for less than $0.5 \%$ of cervical malignancies. The most common presentations are vaginal bleeding, pelvic mass, urinary urgency and constipation. Enlargement of the uterus or cervix can be detected with pelvic examination [5, 6]. However, a differential diagnosis distinguishing between a benign lesion and sarcoma is not possible through history and physical examination. The definitive diagnosis is made by pathological examination. Without a specific pathological finding, it is not easy to diagnose ECSS. In our patient, the diagnosis of ECSS was made by reassessment of pathological specimens after the development of lung metastases. There was no specific sign except for poor focal staining with CD10 in the stroma in the lung and the endocervical tumor. Because of the insignificance of malignancy criteria in the endocervical tumor, the lung metastasis and reassessment of histomorphological signs led to a diagnosis of ECSS.

The prognosis correlates with stage, grade, surgical margins and number of mitoses in cervical and uterine sarcomas. Vascular and lymphatic invasion are negative 
prognostic factors [7]. The choice of treatment in nonmetastatic patient is surgery. Unfortunately, the relapse rate is very high with surgery alone [8]. Due to a small number of cases, there is no survival information or consensus about adjuvant treatment of ECSS. In the study of Reed et al., local recurrence was less frequent in patients who received adjuvant radiotherapy [9]. But there was no disease-free (DFS) or overall survival (OS) difference. Khosla et al. treated a stage IB2 ECSS patient with surgery followed by radiotherapy and chemotherapy; a stage IIIB patient was treated with primary radiotherapy and chemotherapy [10]. Both patients were disease free at follow-up. The choice of treatment in recurrent and metastatic disease is chemotherapy. The most commonly used agents are ifosfamide, doxorubicin, cisplatin, carboplatin and paclitaxel. We also treated our patient with ifosfamide and doxorubicin and achieved partial remission.

To the best of our knowledge, to date there are 17 ECSS cases in the literature. Our patient is the $18^{\text {th }}$ case and the first ECSS patient to develop orbital metastasis. Based on our experience in this case, we may say that although surgery is the mainstay treatment for nonmetastatic ECSS patients, ifosfamide and doxorubicin are active chemotherapeutic agents for ECSS. Radiotherapy may also be effective in symptomatic treatment of these patients.

\section{Disclosure}

Authors report no conflict of interest.

\section{References}

1. Carcangiu ML. Mesenchymal tumours. In: Tavassoli FA (ed.). Pathology and Genetics Tumours of the Breast and Female Genital Organs. IARC Press, Lyon 2003; pp. 280-283.

2. Fadare O, Ghofrani M, Stamatakos MD, et al. Mesenchymal lesions of the uterine cervix. Pathology Case Reviews 2006; 11: 140-152.

3. Gao Z, Ouansafi I, Anderson AE. Endocervical stromal sarcoma. J Obstet Gynaecol 2006; 26: 832-835.

4. Moskovic E, Macsweeney E, Law M, et al. Survival, patterns of spread and prognostic factors in uterine sarcoma - a study of 76 patients. $\mathrm{Br}$ J Radiol 1993; 66: 1009-1015.

5. Bansal S, Lewin SN, Burke WM, et al. Sarcoma of the cervix: Natural history and outcomes. Gynecol Oncol 2010; 118: 134-138.

6. Fadare 0 . Uncommon sarcomas of the uterine cervix: a review of selected entities. Diagn Pathol 2006; 1: 30.

7. Rovirosa A, Ordi J, Ascaso C, et al. Prognostic factors in uterine sarcomas: a 21-year retrospective study at the Clinic and Provincial Hospital of Barcelona. Med Clin (Barc) 1998; 111: 172-176.

8. Benoit L, Arnould L, Cheynel N, et al. The role of surgery and treatment trends in uterine sarcoma. Eur J Surg Oncol 2005; 31: 434-442.

9. Reed NS. The management of uterine sarcomas. Clin Oncol (R Coll Radiol) 2008; 20: 470-478.

10. Khosla D, Gupta R, Srinivasan R, et al. Sarcomas of uterine cervix: clinicopathological features, treatment, and outcome. Int J Gynecol Cancer 2012; 22: 1026-1030. 